\title{
The Network from Above and Below
}

\author{
Pat Brundell, Andy Crabtree, Richard Mortier, Tom Rodden, Paul Tennent, Peter Tolmie \\ School of Computer Science \\ University of Nottingham \\ Nottingham NG8 1BB, UK \\ +441159514251 \\ firstname.lastname@nottingham.ac.uk
}

\begin{abstract}
Recently, the HCI community has taken a strong interest in problems associated with networking. Many of those problems have also been the focus of much recent networking research, e.g., traffic identification, network management, access control. In this paper we consider these two quite different viewpoints of the problems specifically associated with home networking. Focusing on traffic identification as a core capability required by much recent HCI work, we explore the mismatch between the approaches the two communities have taken, and suggest some resulting challenges and directions for future work.
\end{abstract}

\section{Categories and Subject Descriptors}

C.2.3 [Network Operations]: Network monitoring.

\section{General Terms}

Measurement, Design, Human Factors.

\section{Keywords}

Home networks; Application identification; Ethnographic fieldwork.

\section{INTRODUCTION}

The network has become a major empirical focus in HCI. Recent studies have provided empirical insights into the ways in which network technologies are being incorporated into everyday life, e.g., [6][15][18]. Empirical studies have focused on network infrastructure and the work implicated in setting up and maintaining home networks [6], the work of weaving the home network into domestic routines [18], and user understandings of the home network [15]. Many have made the point that the design and construction of these domestic networks are opaque to users, stressing the need to enhance the network's transparency and local accountability, e.g., [3][16].

Studying network use has a tradition within the systems community that significantly predates recent interest by $\mathrm{HCI}$

Permission to make digital or hard copies of all or part of this work for personal or classroom use is granted without fee provided that copies are not made or distributed for profit or commercial advantage and that copies bear this notice and the full citation on the first page. To copy otherwise, or republish, to post on servers or to redistribute to lists, requires prior specific permission and/or a fee.

W-MUST'11, August 19, 2011, Toronto, Ontario, Canada.

Copyright 2011 ACM 978-1-4503-0800-7/11/08...\$10.00. researchers. Network traffic modeling and analysis is a key cornerstone of how the systems and networking communities seek to understand and manage increasingly complex networks. Elaborate models of the nature of different forms of traffic and the temporal characteristics of this traffic have been developed to characterize and explain network behavior e.g., [4][8][12][13].

We suggest that a gulf exists between the ways in which the network is perceived by those who study and understand it from the perspective of users, and by those who analyze and understand traffic from the perspective of the network. Although both seek to understand and characterize the forms of network use and the temporal nature of the network, the characterizations of network traffic and use are markedly different. Mapping between these different perspectives represents a fundamental research challenge.

In this paper we briefly explore these two perspectives before discussing the ways in which they need to be reconciled, and the research agenda that doing so raises for both the HCI and the Systems and Networking communities.

\section{THE VIEW FROM ABOVE}

We wish to begin by considering how the domestic network is seen from the perspective of those who use it, and how they make use of it. Researchers have exploited field studies and humancentered design techniques to develop the community's understanding of home networks. In a seminal paper, The Work To Make The Home Network Work, Grinter et al. [6] subjected the home network to the same kind of careful empirical scrutiny that HCI and CSCW researchers had previously accorded the introduction of networks into the workplace [2]. The study revealed that home networks are characterized by 'coordination challenges' implicated in the management of shared resources, troubleshooting, and network administration.

Tolmie et al. picked up where Grinter et al. left off, carrying out field studies to articulate the cooperative work implicated in the 'domestication' of home networks [17][18]. These studies revealed that home networks are characterized by 'digital plumbing' and 'digital housekeeping', i.e., the cooperative work involved in situating network technologies in the home and the recurrent and ongoing work of maintaining home networks as part of the broader round of domestic routines. 
We illustrate the nature of this relationship between the network and those who use it by considering a network day drawn from one of our recent studies exploring both network usage in a domestic setting, and how people reason about the activities of the network.

\section{A NETWORK DAY}

To gain an understanding of domestic network use we undertook a series of studies that combined fieldwork with a bespoke HTTP proxy logger to record network activities. The studies formed part of a broader ongoing enterprise to understand the impact of the home network on everyday life, involving 8 households located largely in the Midlands area of the UK. The logging tool ran on an Acer eeePC with a solid state hard drive, which was given a static IP address and connected directly to the household's wireless access point via a wired Ethernet connection. Written in Java, the software consisted of a simple multithreaded logging HTTP proxy.

Logging was complemented by fieldwork, which prefaced installation of the proxies and continued intermittently throughout their deployment, which varied according to household permission. Fieldwork focuses on exploiting situated observation of action in context and informal interviews to account for the organization of technology use in the home and to elaborate the nature of the logged activities. The logs informed and directed fieldwork, providing a focus for empirical study and a starting point for considering the use of the network with users.

To illustrate our broader points we focus on the results to emerge from one particular home network. The household in question consisted of two adults, one male 'Andy' aged 43, one female 'Tina' aged 42, and two teenage girls, 'Orla' who is 14 and 'Amy' 16. 'The girls are in full time secondary education. Andy is a teacher. Tina is a health worker. Each family member has irregular working hours, as well as a range of commitments and interests outside the home.

\subsection{The Temporal Rhythms of Use}

A striking characteristic of all our households was the extent to which domestic network use is organized in terms of temporal rhythms and routines that exhibit differences between weekdays and weekends. In the particular household in question, almost all network use on a weekday occurs between $3 \mathrm{pm}$ and midnight, with activity peaking between $7 \mathrm{pm}$ and $10 \mathrm{pm}$. Internet access by adults is primarily concentrated into the hours between $8 \mathrm{pm}$ and $10 \mathrm{pm}$. These distinctive rhythms and routines are driven by the exigencies of everyday life: during the week the girls go to school, the adults to work. As Andy puts it,

"The girls get home at quarter to 4. I get back 6-ish, Tina gets back similar time, sometimes later. In terms of Tina and me it's getting back, pottering about, doing things, cooking food, sorting bits and bobs out, so if we then use the computer it will tend to be after that; kind of 7, half 7, 8 o'clock onwards."

\footnotetext{
${ }^{1}$ Not their real names.
}

Evidently, the exigencies of everyday life extend beyond going to school and work to "pottering about, doing things, cooking food, sorting bits and bobs out"; in other words, that gamut of contingent and routine activities that animate everyday life at home and take priority over Internet use. As Tolmie et al. [18] note with reference to digital housekeeping,

"Many routine activities in the home are given priority: access to the bathroom before going to work, children's bedtime, and so on ... [Consequently, digital housekeeping] becomes something that will fit in, around and with other routines."

The rhythms and routines that might be evident in any network are shaped by other household routines and the priority they have on members' activities. This means that for the adults, network activities are concentrated into short time frames, often during the evening after domestic chores are completed, whereas the teenagers have a period after arriving home from school and before the adults come home when they are relatively unconstrained in their activities. As Andy describes it,

"The girls get home from school an hour, an hour and a half, before we do and then they're installed in there [the living room] with their laptops and TV on."

It is important to appreciate that routines can be quite subtle: they are not necessarily manifest everyday but may be more occasional in their frequency. For example, Amy's weekend network activity occasionally dropped to a much lower level. It turns out that she has an irregular weekend job at a local restaurant, where she works variable shifts, sometimes Friday and Saturday evenings, sometimes also during the day, and occasionally she is not required at all. Furthermore, when not working at the restaurant, Amy is often engaged in extended periods of sporting activity. School, work and play all combine to shape Amy's use of the home network and result in routine periods of intense Internet activity that are fitted around outside commitments.

Understanding the inner life of the network requires that we understand the outer lives of its users as well. Rhythms and routines of use are rooted in and shaped by the social organization of everyday life: in going to school, going to work, playing sports, doing domestic chores, and all the other 'bits and bobs' that need 'sorting'. Network activities reflect the social organization of everyday life; they get their sense and reference from it, as can be seen when we consider service access routines in more detail.

\subsection{The Difference Between Access and Use}

It is worth reflecting on the distinction between network access and the actual use of network services by users. For example, websites increasingly consist of portal services, which are often used in a variety of ways. For example, Facebook may be used for various purposes, from chat to gaming to photo and media sharing. For the majority of the time they are online, Amy and Orla's machines are accessing Facebook. This is not the case for the adults' shared machine, which shows regular but not continuous Facebook access. Similarly, Amy's laptop shows almost continuous access to MSN Messenger, Windows Live, and 
Hotmail services unlike the other machines, which showed regular but intermittent access.

Fieldwork revealed that Amy routinely boots up her machine, signs into MSN, and then clicks on the Hotmail inbox icon. Amy accounts for the procedure in saying,

“... it's like stuff that I've emailed from my school account to my home account, like documents and stuff."

Given this style of work it is unsurprising that Messenger, Live, and Hotmail services appear to be accessed to a high degree. However, the use Amy to which puts these services is not immediately apparent from any logging or measurement. Interaction between her school network account, which leaves a small trace in the logs, and other communication services is hard to infer from the logs alone.

The moral of the story is that inferring continuous service use by household members on the basis of measurement of access by a machine is unwarranted. What is warranted though, is the inference that household members have different work-practices of service use: for example, the adults frequently log in and out of MSN services, pulling data to them on demand; whereas the children adopted an always on practice with data continuously pushed to them.

\subsection{The Diversity of Network Activities}

Our studies also suggest stability about network use that allows people to speak about and characterize the ways in which they use the network. These characterizations are reflected in how they talk about and describe their network use. The characterizations at play tend to focus not on forms of traffic or the application being used but on the activities the network enables and allows. As Tina put it when discussing her use of the web during the logging period,

"I used it like I normally do: the bank, Hotmail and John Lewis [laughs]"

John Lewis is a large department store in the UK. Tina uses John Lewis on a regular basis for her shopping needs. What is interesting here is the everyday language used to characterize different sorts of use. These separations are not technical - for Tina they are based on activity and purpose. Thus she does not use the web, transfer files, fetch email, or stream media; instead, she shops, banks, watches films and sends messages.

Users often use these characterizations of the network to describe how the network is used. For example, Andy offered the following account of Orla's activities during a fieldsite visit in which Orla was quite visibly 'otherwise engaged',

"A lot of it will be headphones, you know, music on the laptopshe listens to Grooveshark - there'll be Facebook, Messenger, one or two other things on at the same time."

Users characterizations' are practical understandings of the network. They may have a loose association with the types of traffic and application in play, but fundamentally what they convey are the different things done with the network. From our broad range of studies we suggest a number of common distinct categories emerge from our work.

Email is often talked about as a particular form of network use. People talk of "getting their email" or "catching up with email" but use a range of applications and protocols for email. These include Outlook/Exchange on Windows, as well as clients using POP3 and IMAP, as well as significant use of various webmail systems from Microsoft, Google, Yahoo and others.

Gaming was a particular class of activity spoken about by users. This was often in terms of the impact of this form of activity on the network. People would talk about "gaming slowing the network" or complaints of others slowing the network stopping gaming.

Skype audio and video calling has grown dramatically over recent years, in both domestic and work settings. ${ }^{2}$ Users often speak of regular video communication and conversations and the impact this has on the overall network performance.

Social media such as Facebook, MSN and Twitter are often referred to as a part of the overall activity of the network. Often this is presented and talked about in terms of access: "you're not allowed on Facebook until you've done your homework." Given the growing popularity of social games, the inter-relationship between this categorization and gaming needs further study.

Streaming media has a growing presence in homes, with services such as BBC iPlayer and Last.fm becoming the normal means of media consumption. For video services, people coordinate with others to ensure video watching is not impacted due to network congestion.

Downloads are a specific set of network activities having a dramatic effect on domestic networks, and were often talked about negatively due to their perceived impact on the home network. People were often asked to schedule any downloads considerately, e.g., asking others to "do your downloads at night time."

Shopping was a specific online activity for many users, spanning a number of websites including both traditional and specifically online retailers, e.g., John Lewis, Amazon. People were concerned that shopping activities would not be impacted by network variations. This was particularly critical when processing final payments or undertaking activities such as comparative browsing of products.

\subsection{Where the Network is Used}

Network use is highly situated, with network activities taking place in particular settings and situations. People routinely reason about and account for what one another are up to on the network, according to their local understanding of those settings and situations; this includes how they understand those activities to be embedded in and exploiting space and place.

Thus, fieldwork makes it perspicuous that the use of network services by the girls is exclusively confined to the family living

2 http://www.techtified.com/2010/05/13-percent-of-internationalcalls-now-go-via-skype/ 
room. On the other hand, adult access occurred exclusively in the kitchen. Though there was regular visual and verbal contact between the girls in the living room and the adults in the kitchen, there was a definite spatial or ecological separation of network service access between adults and teenagers. Typically, this separation maintains until around 9pm when domestic chores have usually been completed and eating has finished. However, when the adults have addressed the day's priorities, they deliberately try to claim the living room space to watch TV, often the BBC's online iPlayer service. As Andy puts it,

"When things settle down for us we'll ask the girls 'what have you got to do?' Orla often goes off to her room to do her homework, Amy normally stays about; she's more sociable."

We are not suggesting that this particular arrangement is of any general significance. We are suggesting that use of network services is intimately connected to the organization of space and place and the ways in which that organization is locally designed to meet social need [6][18]. As Tina puts it,

"We had discussion before we moved in that we wouldn't have television points in the girls' bedrooms because they escaped to their bedrooms too much and I wasn't happy about the amount of time they spent on their own. The laptops don't work in their bedrooms - fantastic! - so they have to be in the sitting room which is where I'd rather it be: a communal thing so there's people about, more social interaction, we get to share more"

In this case what could be viewed as poor performance of the wireless network becomes a concrete benefit! Previous empirical studies have highlighted that household members organize space to cater for network technology, e.g., [6][18]. When we move beyond infrastructure it is also the case that household members organize the spatially embedded use of technology around the social dynamics, expectancies and routines that hold sway in the home. Not only is the positioning of network technology "accountable to the broader issues in the household" [6], its situated use is shot through with them.

This section has explored how network users understand home networks in terms of when they are used, what use is made of them, and where this use takes place. In the following section we consider the understanding of network use "from below", i.e., how this use might be understood from the perspective of the traffic visible on the network.

\section{THE VIEW FROM BELOW}

Traditional network measurement and management techniques arose in large backbone and later enterprise networks. Using extensive and distributed measurement techniques, statistical models are constructed primarily for purposes such as attack detection and capacity planning, but also to describe network behavior to enable simulation of network traffic. These models typically take account of time-of-day, day-of-week, seasonal, and other macroscopic factors leading to temporal variation. They describe traffic in terms of volume (byte and packet counts) and generating application, often aggregated into coarser categories.
Models for attack detection often also consider communication patterns between hosts as identified by IP address.

The application classes and categories into which network engineers classify traffic are based on factors with network-wide significance. For example, large shifts in traffic volume signifying rerouting events due to, e.g., link failure; changes in the applications generating the bulk of traffic indicating trends pertinent to capacity planning, e.g., the changing significance of email, web and peer-to-peer file sharing traffic over the past decade; and communication patterns indicative of security scans, attacks and breaches whether low-volume port-scanning or high volume targeted distributed denial-of-service.

Unfortunately, these traditional categorizations are of limited use in the home network context. Not only do the events of significance change when considered from a home network point of view, even from a network point-of-view these categories are not always cleanly separated. For example, others have noted the rise of HTTP as a universal transport protocol, used for web browsing, social communications, video access and email, often muddies the waters [10][14].

Additionally, applications that generate low volumes of traffic across a backbone network might be considered highly significant by the homeowner when carried on their home network. For example, Skype traffic is not typically such high volume that it is of particular importance to the network operator. However, as noted above, to the homeowner, how Skype traffic is treated may well be critical to their work and social lives.

\subsection{Categorizations}

There is no explicit consensus in the networking community as to how to categorize traffic by application, with a range of ad hoc categorizations and many authors even using the terms "application" and "protocol" interchangeably, e.g., [1][4][8]. The nearest to a consensus view appears to be that of Moore et al. [12][13], recently updated by Kim et al. [9]. The resulting categorization is given in Table 1, with some less widely used extensions given in Table 2.

Comparing these to the categories from users (§3.3) we see limited overlap, e.g., gaming, streaming, email, downloads (P2P). As other authors have noted however, some of these categories are misleading, e.g., email now really must include some web traffic due to widespread use of Hotmail, Gmail and others. One category beloved of network engineers that is notably absent from the user categorization is that of the Web (WWW). It seems that, perhaps unsurprisingly, users categorize their network behavior by activity not by protocol or even service. Thus the network engineer's "www" becomes a mixture of (in this case) "social media", "streaming media", "email", and "shopping". It is quite possible that a broader sample of users would further extend that category.

Skype deserves special mention as a category important to users: it is difficult to identify, designed to evade firewalls and NATs, and to obfuscate its traffic patterns. Furthermore, it uses both TCP and UDP, and in some cases uses well-known ports $(80,443)$. 
Considerable effort has been expended to identify Skype nonetheless, perhaps because its presence often poses a threat to traditional network operator profit models. A variety of techniques have been used; perhaps the most thorough approach is that of Molnár [11] which makes use of observed signaling traffic on a range of ports, timing characteristics of UDP keepalive messages, and a range of call connection properties (bandwidth, packet rate, average packet size, main mode of packet inter-arrival time).

Table 1. Consensus network traffic categorization [9].

\begin{tabular}{ll}
\hline Application & \multicolumn{1}{c}{ Protocols } \\
\hline WEB & HTTP, HTTPS \\
\hline P2P & $\begin{array}{l}\text { FastTrack, eDonkey, BitTorrent, Ares, Gnutella, } \\
\text { WinMX, OpenNap, SoulSeek, MP2P, Direct } \\
\text { Connect, GoBoogy, Soribada, PeerEnabler }\end{array}$ \\
\hline FTP & FTP \\
\hline DNS & DNS \\
\hline Mail/News & SMTP, POP2/3, IMAP, identd, NNTP \\
\hline Streaming & MMS (Windows Media Player), Real, Quicktime, \\
& Shoutcast, VBrick streaming, Logitech Video IM \\
\hline Network & NetBIOS, SMB, SNMP, NTP, SpamAssassin \\
operations & GoToMyPc \\
\hline Encryption & SSH, SSL \\
\hline Games & Quake, HalfLife, Age of Empires, Battlefield \\
\hline Chat & Vietnam \\
\hline Attack & AIM, IRC, MSN Messenger, Yahoo messenger \\
\hline Unknown & \\
\hline
\end{tabular}

Table 2. Additional commonly used categories [5][7].

\begin{tabular}{ll}
\hline Application & \multicolumn{1}{c}{ Protocols } \\
\hline VoIP & $\begin{array}{l}\text { SIP, RTP, Cisco Callmanager, SCCP, Vocera, } \\
\text { Skype }\end{array}$ \\
\hline Filesystems & SMB, CIFS, NetBIOS, Appleshare, NFS, AFS \\
\hline Services & X11, DNS, finger, identd, DND, Kerberos, \\
& LDAP, NTP, printer \\
\hline
\end{tabular}

\section{IMPLICATIONS \& CHALLENGES}

Having discussed these two rather disparate views of network traffic classification, the question arises: so what? In this section we present and discuss some of the implications and challenges that arise in reconciling them.

Legibility. As network connectivity becomes more ubiquitous in household devices, e.g., TVs and fridges, network management becomes a required everyday activity. However, those that must carry it out are unlikely to want to become professional network managers, thus it behooves the networking community to provide better, more accessible tools to give greater insight into the network to non-experts, who do have no desire to concern themselves with details such as protocols. Traffic classification for presentation in user interfaces must take account of the categories that are important to users.

Quality of service (QoS). The major sites of congestion are currently believed to be the home and edge networks. QoS mechanisms can be used to make better use of available resource, but must be applied to the service not the protocol. Thus it might be video (not RTMP), or Skype (not VoIP), or shopping (not HTTP) that needs protection. Doing so relies on both accurate identification of traffic with useful categories, and automatic techniques for determining the bandwidth requirements and impact of any given QoS mechanism.

Temporality. The high degree of temporal variation in both traffic volume and type, coupled with the fuzziness and complexity with which related policies are expressed, e.g., "no Facebook until after dinner", "no gaming except at weekends", gives rise to and scope for mechanisms to timeshift different types of traffic. Taking these factors into account might inform operators' capacity planning, as well as prove useful in alleviating particular hotspots. The presence of activity from certain applications, devices or even users on the network might be used to indicate the temporal rhythm of the setting allowing different policies should come into play at key times.

Flexibility. Home networks evolve the types of traffic carried and the demands placed upon them more quickly and more radically than core networks. The simple addition of a new device such as the latest games console can significantly alter the traffic patterns observed on a home network, as well as radically change the relative desired treatment of traffic by the network. Classification or management tools for home networks must be sufficiently flexible to cope with these step changes.

Geography. Home network access and use is socially mediated, to a far greater degree than in the enterprise network. Even given the reliance on wireless networking technologies, the geography of the home still plays an important part in this. How well signals propagate through the physical building has an important impact on network use from different points: how public or private the situation will change both permitted and actual behavior.

\section{ACKNOWLEDGMENTS}

The research on which this paper is based was funded by the RCUK supported Horizon Hub, EP/G065802/1, and EPSRC Wired and Wireless Intelligent Networked Systems Initiative 'Homework', EP/F064276/1.

\section{REFERENCES}

[1] Bernaille, L., Teixeira, R., and Salamatian, K. "Early application identification", Proceedings of ACM CoNEXT, '06.

[2] Bowers, J. and Rodden, T. (1994) "The work to make a network work: studying CSCW in action", Proceedings of CSCW '04, pp. 287-298, Chapel Hill, ACM.

[3] Chetty, M., Banks, R., Harper, R., Regan, T., Sellen, A., Gkantsidis, C., Karagiannis, T. and Key, P. (2010) "Who's hogging the bandwidth? The consequences of revealing the 
invisible home", Proceedings of CHI '10, pp. 659-668, Atlanta, ACM.

[4] Crotti, M., Dusi, M., Gringoli, F., and Salgarelli, L. "Traffic Classification Through Simple Statistical Fingerprinting", ACM SIGCOMM Computer Communications Review 37(1) January 2007.

[5] Erman, J., Gerber, A., Hajiaghayi, M.T., Pei, D., and Spatscheck, O. "Network-aware forward caching", Proceedings of $A C M W W W$ ' 09 .

[6] Grinter, R., Edwards, K., Newman, M. and Duchenaut, N. (2005) "The work to make the home network work", Proceedings of ECSCW '05, pp. 469-488, Paris, Springer.

[7] Henderson, T., Kotz, D., and Abyzov, I. "The Changing Usage of a Mature Campus-wide Wireless Network", Proceedings of ACM MOBICOM '04.

[8] Iliofotou, M., Pappu, P., Faloutsos, M., Mitzenmacher, M., and Varghese, G. "Network Monitoring Using Traffic Dispersion Graphs", Proceedings of IMC '07. ACM.

[9] Kim, H-C., Claffy, kc., Fomenkov, M., Barman, D., Faloutsos, M., and Lee, K.-Y. "Internet Traffic Classification Demystified: Myths, Caveats and the Best Practices", Proceedings of ACM CoNEXT' 08 .

[10] Maier, G., Feldmann, A., Paxson, V., and Allman, M. (2009) "On Dominant Characteristics of Residential Broadband Internet Traffic”, Proceedings of IMC '09. Chicago, Illinois, USA. ACM.
[11] Molnár, S. and Perényi, M. "On the identification and analysis of Skype traffic", International Journal of Communication Systems, 24(1):94-117, January 2011.

[12] Moore, A.W., and Konstantina, P. "Toward the Accurate Identification of Network Applications", Proceedings of $P A M$ '05. LNCS 3431, 2005.

[13] Moore, A. and Zuev, D. "Internet Traffic Classification Using Bayesian Analysis Techniques", Proceedings of ACM SIGMETRICS '05.

[14] Schneider, F., Agarwal S., Alpcan T., and Feldmann A. (2008) "The New Web: Characterizing AJAX Traffic", Proceedings of PAM '08.

[15] Shehan Poole, E., Chetty, M., Grinter, R., Edwards, K. (2008) "More than meets the eye: transforming the user experience of home network management", Proceedings of DIS '08, pp. 455-464, Cape Town, ACM.

[16] Tolmie, P. (2010) "Making the home network accountable", 2010 Multi-Service Networks Workshop, July 8-9, Cosener's House, Abingdon, UK.

[17] Tolmie, P., Crabtree, A., Egglestone, S., Humble, J., Greenhalgh, C. and Rodden, T. (2010) "Digital plumbing: the mundane work of deploying ubicomp in the home", Personal and Ubiquitous Computing, vol. 14 (3), pp. 181196.

[18] Tolmie, P., Crabtree, A., Rodden, T., Greenhalgh, C. and Benford, S. (2007) "Making the home network at home: digital housekeping”, Proceedings of ECSCW '07, pp, 331350 , Springer. 